\title{
Hybrid Manta Ray Foraging Optimization for Novel Brain Tumor Detection
}

\author{
Dr. P. Karuppusamy, \\ Professor, \\ Department of EEE, \\ Shree Venkateshwara Hi-Tech Engineering College, \\ Erode, India. \\ Email: pkarrupusamy@ieee.org
}

\begin{abstract}
In medical image processing, segmentation and extraction of tumor portion from brain MRI is a complex task. It consumes more time and human effort to differentiate the normal and abnormal tissue. Clinical experts need more time to provide accurate results, recent technology developments in image processing reduces the human effort and provides more accurate results which reduces time and death rates by identifying the issues in early stage itself. Machine learning based algorithms occupies a major role in bio medical image processing applications. The performance of machine learning models is in satisfactory levels, but it could be improved by introducing optimization in feature selection stage itself. The research work provides a hybrid manta ray foraging optimization for feature selection from brain tumor MRI images. Convolution neural network is used to test the optimized features and detects the early stage brain tumors. The experimental model is compared with existing artificial neural network, particle swarm optimization algorithm and acquires a better detection and classification accuracy.
\end{abstract}

Keywords: - Magnetic Resonance Image, Manta Ray Foraging Optimization, Convolution Neural Network

\section{Introduction}

Brain tumor is an important mortal type cancer and it affects the neurons in human and leads into serious issues. So, it is essential to identify the tumors in early stage itself to avoid major health issues and sometimes death. Researchers are still working on it to develop a mechanism to identify the early stage detection and the research still goes on. Early detection will increase the possibility of curing the disease and save patients life. The survival possibility of early stage detected patients are high compared to late detection. Figure 1 depicts the various types of brain tumors.

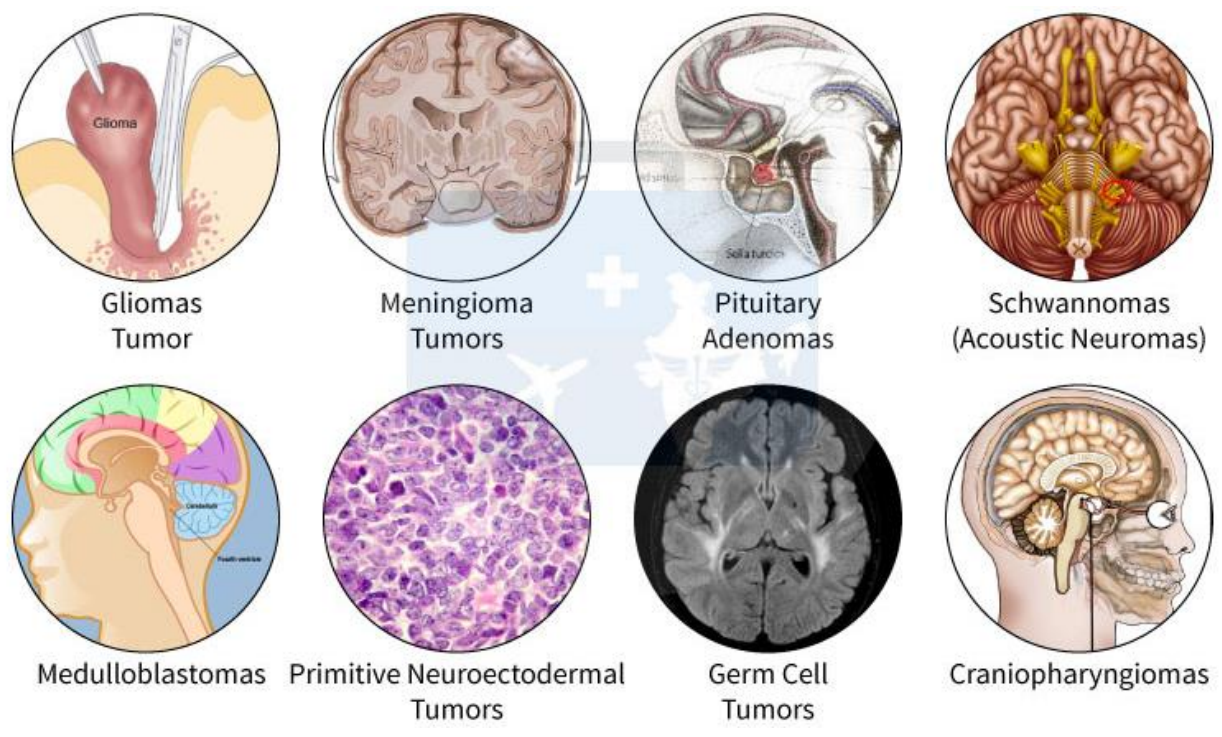

Fig.1 various types of Brain Tumors

Brain tumor simply referred into accumulation of cells in the brain, these cells are called as abnormal cells which is different from existing brain cells. Generally, these abnormal cells grow inside the skull so that it starts 
forcing the normal cells and leads into serious pain and affects the normal function of brain. Tumors are classified into two types such as

- $\quad$ Benign Tumor- It is an early stage tumor which could be non-cancerous.

- Malignant Tumor- It is the last stage tumor which produces dangerous effects.

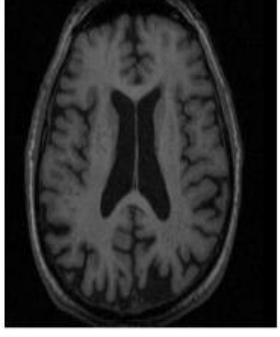

(a)

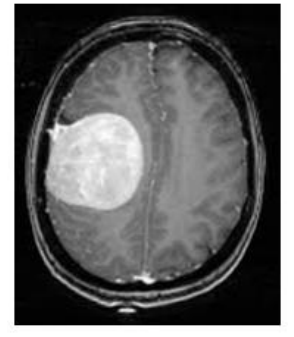

(b)

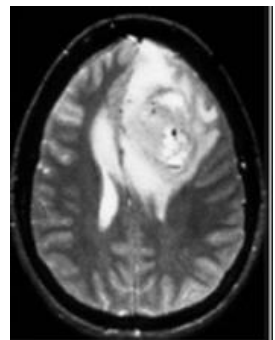

(c)

Fig.2 (a) Normal Brian Image (b) Benign Tumor (c) Malignant Tumor

Figure 2(a) depicts the Normal MRI image of human brain and figure 2(b) represents the benign tumor image, figure 2(c) depicts the malignant tumor. Based on the position and nature of tumor, it is classified into two categories such as primary tumor which is located in the brain and it comes under benign type. The secondary tumor is originated from any part of the body and spreads into brain which is comes under malignant type. Figure 3 depicts the process flow of brain tumor medical image processing. The process starts with the MRI input image, which is further pre-processed to remove the noise and other features. Followed by pre-processing, the features are selected through proper segmentation process. Once the features are selecting using classifier algorithms the results are obtained.

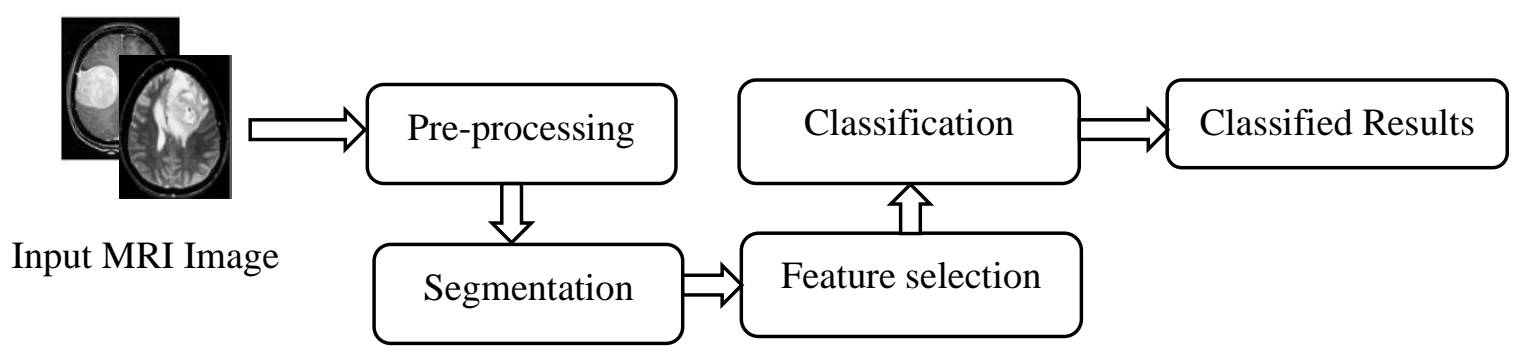

Fig.3 Conventional Brain Tumor Detection

Early detection increases the survival probability and reduces the death risks. However early detection requires more human efforts and experts to recognize the tumor and it costs more which is not afforded and applicable to all people. This leads into implementation of computer aided detection mechanisms to detect brain tumor automatically through software applications. Technology development helps in predicting the early state of tumors and assist the experts by helping with probability reports. Researchers are still working on it to improve the detection efficiency of the early stage detection. For this various application are developed in various times and in the next section the methods and its merits and demerits are discussed to obtain the motivation of the proposed tumor detection model.

\section{Related works}

A vast survey has been made for identifying the issues in existing brain tumor detection models. In order to obtain the details about various techniques. Mahmoud Khaled Abd-Ellah et.al.[1] survey has been used. In this various conventional and recent techniques are analyzed for brain tumor detection. Along with this an intense survey is made with the mentioned techniques to obtain the problem gap in the existing models. Devkota et.al.[2] reported the issue in brain tumor detection through morphological operation to obtain better performance in computation time. Since the main factor to move on with computer aided designs are to reduce the computation 
time. The proposed model attains better computation time but lags in the performance on its evaluation stage. The proposed model reaches an accuracy of $92 \%$.

Similar morphological operations like threshold-based segmentation Umit Ilhan et.al [3], pixel subtraction Gamal.et.al [4] and filtering techniques Sanjeev Kumar et.al [5] are used to improve the detection accuracy of the tumor portion. In this pixel subtraction is used in various research works for extracting the skull portion from the brain image in an efficient way. Along with pixel subtraction, thresholding approach is used to improve the detection performance. Solmaz Abbasi et.al [6] discussed the issues in brain tumor detection through histogram-based segmentation. Segmentation plays a vital role in image feature extraction and classification the proposed histogram-based segmentation achieved better classification efficiency. The proposed model differentiates the tumor, edema and normal tissue and an active contour is used to identify the abnormal tissue. Similarly contrast enhancement model is used in Todeschi et.al.[7] research model to identify the tumor portions with increased accuracy of $93.4 \%$. The proposed model efficiency is better but it lags in computation time.

Iván Cabria.et.al.[8] proposed a detection methodology using contrast enhancement which is achieved through k-means method to obtain better performance in terms of specificity and sensitivity. The proposed model attains better efficiency through its simple processing steps with sensitivity rate of $90.2 \%$. Various segmentation methods are introduced to improve the performance in brain tumor detection. Bindhu et.al.[9] proposed Semantic Segmentation method along with adaptive thresholding to define the region of interest in biomedical images. Based on the levels and its characteristics features the tumor regions are identified in the proposed model. Manoharan et.al.[10] proposed a hybrid watershed segmentation algorithm through thresholding approach to reduce the computation complexity. Using morphological operations, the proposed model enhances the quality of the image and segmentation is performed using watershed threshold-based algorithm which provides better classification accuracy with minimum time consumption.

The performance of convention models is satisfactory but the accuracy results are varied while handling few cases. To improve the accuracy, various classification algorithms are introduced in brain tumor detection. ShaoPeng et.al [11] used k-means and support vector machine to develop an automated detection model. The proposed model uses patterns to obtain the features. Based on the features along with support vector machine, skull masking is combined to define the relation between the normal and abnormal images and identifies the tumor region in the proposed model. To improve the system performance few research works uses neuro fuzzy combination or support vector machine which greatly reduces the computation complexity and increases the task performance. Bashar et.al [12] proposed a probabilistic neural network model through vector quantization to improve the processing time and classification accuracy. The proposed model is evaluated and testing using gaussian filter for image smoothening and improves the accuracy. Alexander Zotin et.al.[13] proposed a fuzzy clustering approach to improve the classification accuracy. The decision-based approach identifies the tumor regions and provides better detection results compared to conventional models.

Machine learning models are widely used in brain tumor detection. Javaria Amin et.al.[14] reported the issues in conventional brain tumor detection methods and proposed an improved probabilistic neural network model to reduce the processing time. The proposed model attains better training and test efficiency. Regression based neural networks Yuanpu Xie et.al.[15], deep learning neural network Heba Mohsen.et.al.[16] convolution neural network Saddam Hussain et.al.[17], artificial neural network Philipp Kickingereder.et.al.[18] are introduced various parameters like classification accuracy, computation time, detection efficiency and to reduce the system complexity. In few research models. similarly, optimization algorithms improve the detection accuracy in brain tumor analysis. Various optimization algorithms such as genetic algorithm Rajesh Chandra et.al.[19], particle swarm optimization algorithm Muhammad Sharif et.al.[20], are used to improve the segmentation and classification efficiency. Genetic algorithm improves the performance of system through genomic optimization characteristics which is widely used in various image processing applications. Similarly, particle swarm optimization obtains optimal solution for the issues through its swarm nature. In case of tumor detection these optimization algorithms provide better efficiency compared to conventional morphological operations. Recently ant colony optimization, artificial bee colony optimization, Rabab Hamed et.al.[21] is widely used in image processing applications. Table 1 provides a summary of various research works in detail. 
Table 1 Summary of Existing Research Models in Brain Tumor Detection

\begin{tabular}{|c|c|c|c|c|}
\hline Reference article & Methodology & Merits & Demerits & $\begin{array}{c}\text { Accuracy } \\
\%\end{array}$ \\
\hline Devkota et.al.[2] & $\begin{array}{l}\text { Morphological } \\
\text { Operations }\end{array}$ & $\begin{array}{c}\text { Better Computation } \\
\text { Time } \\
\text { Accuracy } \\
\end{array}$ & $\begin{array}{c}\text { System } \\
\text { Complexity }\end{array}$ & $92 \%$ \\
\hline Umit Ilhan et.al [3], & $\begin{array}{l}\text { Threshold-Based } \\
\text { Segmentation }\end{array}$ & Improved Detection & $\begin{array}{l}\text { Computation } \\
\text { Time }\end{array}$ & $91 \%$ \\
\hline Gamalet.al [4] & Pixel Subtraction & Better Classification & $\begin{array}{c}\text { Computation } \\
\text { Time }\end{array}$ & $90 \%$ \\
\hline Sanjeev Kumar et.al [5] & $\begin{array}{c}\text { Filtering } \\
\text { Techniques }\end{array}$ & $\begin{array}{c}\text { Improved Classification } \\
\text { and Detection } \\
\text { Efficiency } \\
\end{array}$ & $\begin{array}{l}\text { More Parameters } \\
\text { Are to Process the } \\
\text { Image } \\
\end{array}$ & $89 \%$ \\
\hline Solmaz Abbasi et.al [6] & $\begin{array}{c}\text { Histogram-Based } \\
\text { Segmentation }\end{array}$ & $\begin{array}{c}\text { Better Computation } \\
\text { Time } \\
\end{array}$ & $\begin{array}{c}\text { System } \\
\text { Complexity } \\
\end{array}$ & $92.1 \%$ \\
\hline Todeschi et.al.[7] & $\begin{array}{c}\text { Contrast } \\
\text { Enhancement } \\
\text { Model }\end{array}$ & $\begin{array}{c}\text { Classification } \\
\text { Efficiency }\end{array}$ & $\begin{array}{l}\text { Computation } \\
\text { Time }\end{array}$ & $93.4 \%$ \\
\hline Iván Cabria.et.al.[8] & K-Means & $\begin{array}{l}\text { Classification } \\
\text { Efficiency }\end{array}$ & $\begin{array}{c}\text { Lags in } \\
\text { Performance for } \\
\text { Noisy Images } \\
\end{array}$ & $94 \%$ \\
\hline Bindhu.et.al.[9] & $\begin{array}{c}\text { Semantic } \\
\text { Segmentation } \\
\end{array}$ & $\begin{array}{c}\text { Simple and Better } \\
\text { Detection }\end{array}$ & $\begin{array}{l}\text { Computation } \\
\text { Time }\end{array}$ & $92 \%$ \\
\hline Manoharan et.al.[10] & $\begin{array}{l}\text { Hybrid Watershed } \\
\text { Segmentation }\end{array}$ & $\begin{array}{l}\text { Accuracy and Reduced } \\
\text { Computation Time }\end{array}$ & $\begin{array}{c}\text { Needs More } \\
\text { Parameters to } \\
\text { Process } \\
\end{array}$ & $91 \%$ \\
\hline ShaoPeng et.al.[11] & $\begin{array}{l}\text { Support Vector } \\
\text { Machine }\end{array}$ & $\begin{array}{c}\text { Computation Time, } \\
\text { Classification } \\
\text { Efficiency }\end{array}$ & $\begin{array}{c}\text { Lags in } \\
\text { Performance for } \\
\text { Noisy Images } \\
\end{array}$ & $94.5 \%$ \\
\hline Bashar et.al.[12] & $\begin{array}{c}\text { Probabilistic } \\
\text { Neural Network }\end{array}$ & $\begin{array}{c}\text { Classification } \\
\text { Efficiency, Accuracy }\end{array}$ & $\begin{array}{c}\text { Computation } \\
\text { Time } \\
\end{array}$ & $95 \%$ \\
\hline Zotin et.al.[13] & $\begin{array}{c}\text { Fuzzy Clustering } \\
\text { Approach }\end{array}$ & $\begin{array}{c}\text { Accuracy and } \\
\text { Computation Time, }\end{array}$ & $\begin{array}{c}\text { System } \\
\text { Complexity }\end{array}$ & $95.2 \%$ \\
\hline Yuanpu Xie et.al.[15] & $\begin{array}{l}\text { Regression Based } \\
\text { Neural Networks }\end{array}$ & $\begin{array}{c}\text { Classification } \\
\text { Efficiency, Accuracy }\end{array}$ & $\begin{array}{l}\text { Computation } \\
\text { Time }\end{array}$ & $95.1 \%$ \\
\hline Heba Mohsen.et.al.[16] & $\begin{array}{l}\text { Deep Learning } \\
\text { Neural Network }\end{array}$ & $\begin{array}{c}\text { Accuracy and } \\
\text { Computation Time, }\end{array}$ & $\begin{array}{c}\text { System } \\
\text { Complexity }\end{array}$ & $95.6 \%$ \\
\hline $\begin{array}{c}\text { Saddam } \\
\text { Hussain.et.al.[17], }\end{array}$ & $\begin{array}{c}\text { Convolution } \\
\text { Neural Network }\end{array}$ & $\begin{array}{c}\text { Classification } \\
\text { Efficiency, Accuracy }\end{array}$ & $\begin{array}{l}\text { Computation } \\
\text { Time }\end{array}$ & $96.4 \%$ \\
\hline $\begin{array}{c}\text { Philipp } \\
\text { Kickingereder.et.al.[18] }\end{array}$ & $\begin{array}{l}\text { Artificial Neural } \\
\text { Network }\end{array}$ & $\begin{array}{c}\text { Classification } \\
\text { Efficiency, Computation } \\
\text { Time, Accuracy } \\
\end{array}$ & $\begin{array}{c}\text { System } \\
\text { Complexity }\end{array}$ & $96.8 \%$ \\
\hline Rajesh Chandra.et.al.[19] & Genetic Algorithm & $\begin{array}{c}\text { Classification } \\
\text { Efficiency, Computation } \\
\text { Time, Accuracy }\end{array}$ & $\begin{array}{c}\text { Requires More } \\
\text { Parameters to } \\
\text { Process the Input } \\
\text { Data }\end{array}$ & $97.2 \%$ \\
\hline $\begin{array}{c}\text { Muhammad } \\
\text { Sharif.et.al.[20] }\end{array}$ & $\begin{array}{l}\text { Particle Swarm } \\
\text { Optimization } \\
\text { Algorithm }\end{array}$ & $\begin{array}{c}\text { Classification } \\
\text { Efficiency, Computation } \\
\text { Time, Accuracy }\end{array}$ & $\begin{array}{c}\text { Requires More } \\
\text { Parameters to } \\
\text { Process the Input } \\
\text { Data }\end{array}$ & $97.5 \%$ \\
\hline Rabab Hamed.et.al.[21] & $\begin{array}{l}\text { Ant Colony } \\
\text { Optimization }\end{array}$ & $\begin{array}{c}\text { Classification } \\
\text { Efficiency, Computation } \\
\text { Time, Accuracy }\end{array}$ & $\begin{array}{c}\text { Requires More } \\
\text { Parameters to } \\
\text { Process the Input } \\
\text { Data }\end{array}$ & $96 \%$ \\
\hline
\end{tabular}


From the survey it is observed that, conventional models are better in classification and detection performance of brain tumor image. But these models are facing issues in handling noisy images and requires more time to compute. Later neural network models are better in classification accuracy, but it requires an efficient feature selection tool to improve the classification performance. Optimization models are better in obtaining optimal solution for brain tumor detection. Though the performance could be improved by combined with neural network models. based on these limitations in the existing system, the proposed model is designed to achieve better detection and classification performance as a hybrid approach for efficient brain tumor detection.

\section{Proposed Optimization Model}

The proposed optimization model is developed based on the fancy marine creature Manta rays which has flat body with pectoral fins. It swims like a free bird in water. It has a cephalic lobe before the mouth without sharp teeth and feed on microscopic animals in the water. In the foraging process, water is funneled through the mouth and filtered using gills to obtain the food. Generally, it is divided into two categories as reef manta ray and giant manta ray. Manta rays consumes large amount of plankton, on an average adult manta could consume five to ten $\mathrm{kgs}$ of plankton per day. Though the ocean is full of rich resources, but it is evenly dispersed in some regions. Manta rays are very clever in identifying the food resource through its foraging behavior and they travel mostly as groups to find the best place for food resource. The foraging strategy is divided into three process as chain foraging, Cyclone foraging and Somersault foraging.

In chain foraging the manta rays identifies and observes the plankton and reaches to the high concentration region which is considered as better position. The best position is still unidentified, for this the optimization assumes the plankton high concentration to be consumed by manta ray so that it will start to move towards the next best position. In every iteration the individuals on the initial position update its position as best solution until it reaches the final point. The mathematical representation of this chain foraging is given as

$$
y_{i}^{n}(t+1)=\left\{\begin{array}{c}
y_{i}^{n}(t)+r \cdot\left(y_{\text {best }}^{n}(t)-y_{i}^{n}(t)\right)+\sigma \cdot\left(y_{\text {best }}^{n}(t)-y_{i}^{n}(t)\right) \text { for } i=1 \\
y_{i}^{n}(t)+r \cdot\left(y_{i-1}^{n}(t)-y_{i}^{n}(t)\right)+\sigma \cdot\left(y_{\text {best }}^{n}(t)-y_{i}^{n}(t)\right) \text { for } i=2, \ldots M
\end{array}\right.
$$

where $y_{i}^{n}(t)$ is the position of $i t h$ individual in $n t h$ dimension at time $t$ and $r$ is the random vector. Figure 3 depicts the chain foraging process in manta ray optimization.

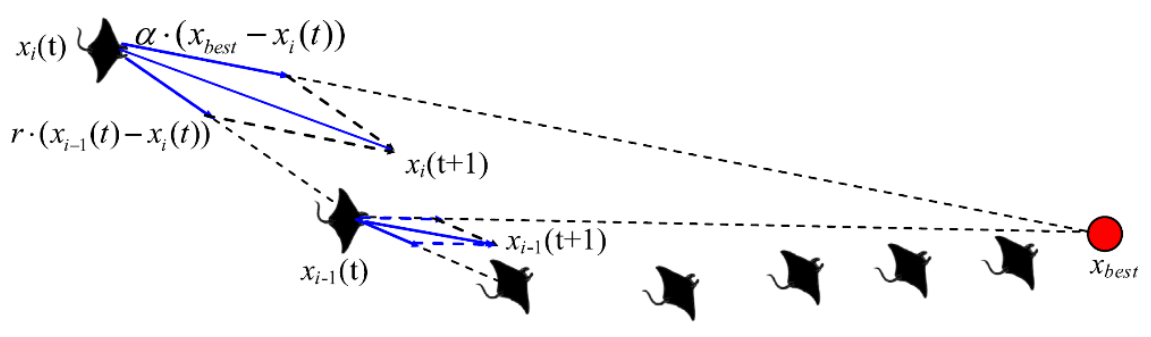

Fig. 4 Chain Foraging in Manta ray optimization

Cyclone foraging is the second step in the manta ray optimization where the manta rays creates a long foraging chain and moves to search foods in a spiral manner. In addition, with cyclone foraging, each manta ray swim towards the other manta ray and develop a spiral structure. It helps the individual to move in front of others and also allows to search food along with the spiral path. The mathematical representation of spiral path is given as follows

$$
\begin{aligned}
& y_{i}^{n}(t+1) \\
& x_{i}^{n}(t+1)
\end{aligned}=\left\{\begin{array}{c}
y_{\text {best }}+r \cdot\left(y_{\text {best }}^{n}(t)-y_{i}^{n}(t)\right)+\cos (2 \pi \omega)\left(y_{\text {best }}^{n}(t)-y_{i}^{n}(t)\right) \text { for } i=1 \\
x_{\text {best }}+r \cdot\left(y_{i-1}^{n}(t)-y_{i}^{n}(t)\right)+\sin (2 \pi \omega)\left(y_{\text {best }}^{n}(t)-y_{i}^{n}(t)\right) \text { for } i=2, \ldots M
\end{array}\right.
$$

where $\omega$ is the random numer in the range $[0,1]$ and the process of cyclone foraging is depcicted in figure 5 . 


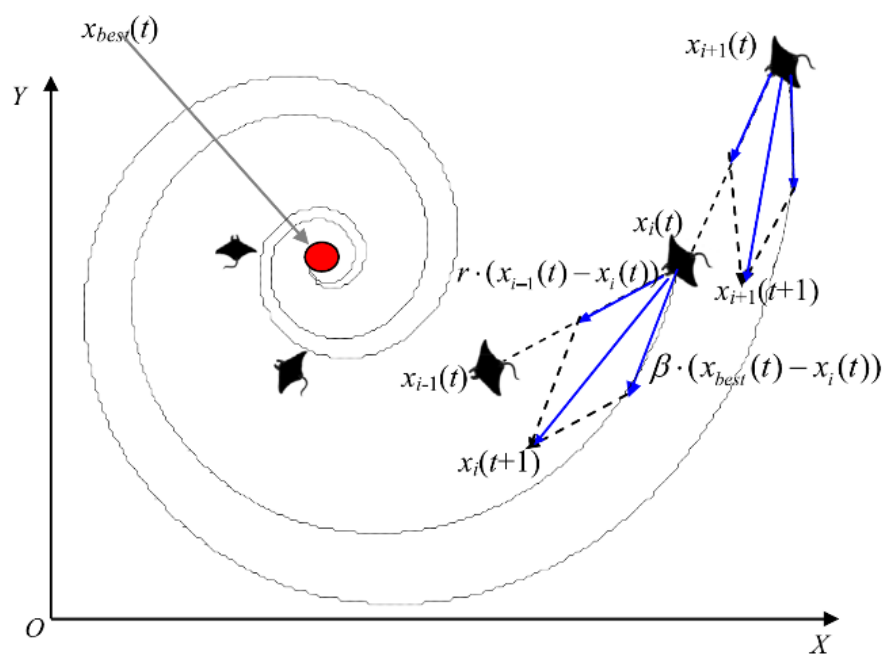

Fig.5 Cyclone Foraging Process

Somersault foraging is the final process in the optimization where the food location is viewed and each individual moves to and fro around the location and somersault in the new position. This helps to update the best position around others. Mathematically it is expressed as

$$
y_{i}^{n}(t+1)=y_{i}^{n}(t)+S \cdot\left(r_{2} \cdot y_{\text {best }}^{n}-r_{3} \cdot y_{i}^{n}(t)\right) \text { for } i=1,2 \ldots . M
$$

where $\mathrm{S}$ is the factor which decides the range of manta ray and $r_{2}, r_{3}$ are the random numbers of range [0,1]. From the equations it is observed that the position of each individual is moved to new position and this process continues until it reaches the best position. Figure 6 depicts the somersault foraging in manta ray optimization.

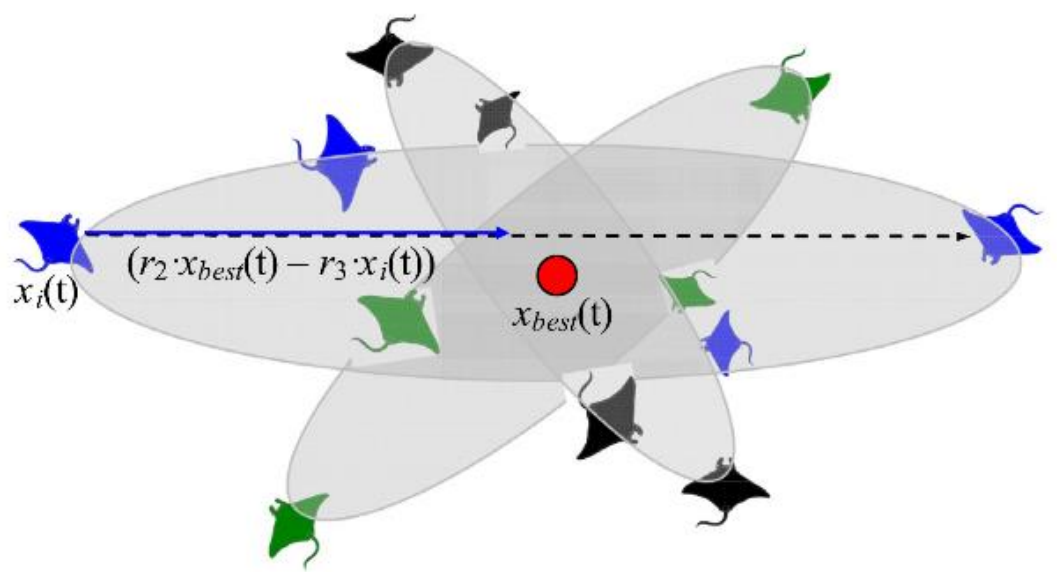

Fig.6 Somersault Foraging in manta ray optimization

Based on this optimization model the proposed brain tumor detection is incorporated to obtain better detection performance. To improve the performance of manta ray optimization, differential evaluation is included to calculate the fitness function. Figure 7 depicts the proposed model process diagram for optimized tumor detection. 


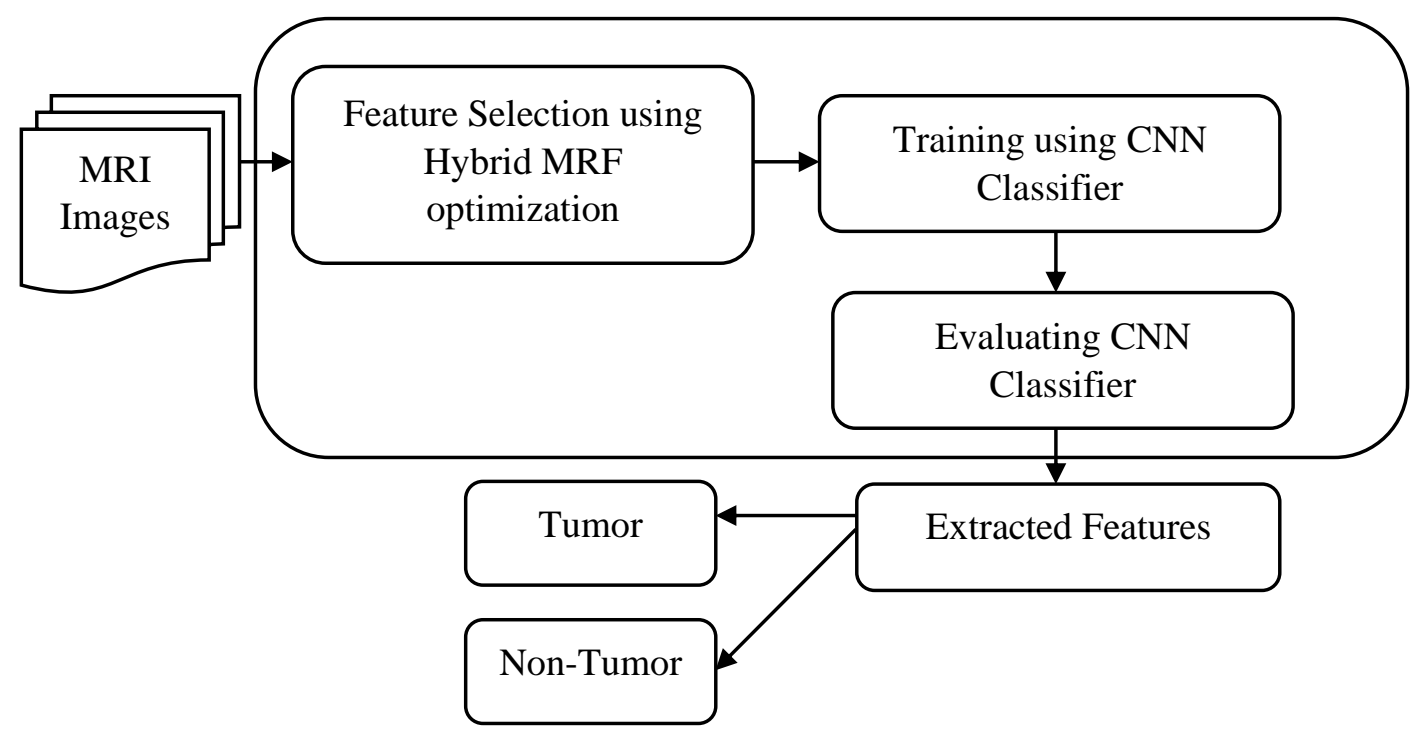

Fig. 7 Proposed Model Process flow

The process starts with MRI Brain image and the features are selected using hybrid manta ray foraging optimization. Once the features are selected it is trained using convolution neural network which is attain better performance over other neural network architectures. It classifies the data and evaluates the classifier results based on the extracted features of classifier the final results are obtained for tumor or non-tumor images. The proposed model used differential evaluation to calculate the fitness function. This calculated fitness functions are computed and it indicates the quality of the selected feature which further improves the performance of manta ray foraging optimization. The best position is selected based on the best agent and the positions are updated based on the fitness function which is different from the conventional manta ray optimization. The steps used in the proposed model is summarized as follows

Step 1. Select the features based on the manta ray foraging optimization

Step 2. Calculate the fitness function for each selected feature and proceed the data into classifier.

Step 3. Proceed the data with CNN classifier and select operational data

Step 4. Select the features and convert into training data to obtain better results.

Step 5. Calculate the accuracy of classification process until the iteration reaches the best position in identifying the issue.

Step 6. Repeat step 1-5 for rest of the features and terminate the process if all the features are processed.

\section{Result and Discussion}

The proposed model manta ray optimization is simulated in MATLAB optimization toolbox with dataset of 500 MRI Brain images. In these 250 images are benign tumors and 150 are malignant tumor images and the rest of the images are normal brain images. The performance of proposed model is parallelly compared with conventional neural network models such artificial neural network and particle swarm optimization algorithm. The fitness value of each process is carefully observed and listed in table 2 . 
Table 2. Fitness values comparison

\begin{tabular}{|c|c|c|c|c|}
\hline S.No & Parameters & ANN & PSO & Proposed MRFO \\
\hline 1 & Mean & 0.1292 & 0.0359 & 0.0262 \\
\hline 2 & standard deviation & 0.0072 & 0.0046 & 0.0028 \\
\hline 3 & best & 0.1291 & 0.1242 & 0.0165 \\
\hline 4 & worst & 0.0426 & 0.0356 & 0.0314 \\
\hline
\end{tabular}

The average of selected features is depicted in figure 8. It is observed that the proposed optimization model has better score compared to other models. The three-stage foraging process improves the feature selection in the proposed model and attain better performance.

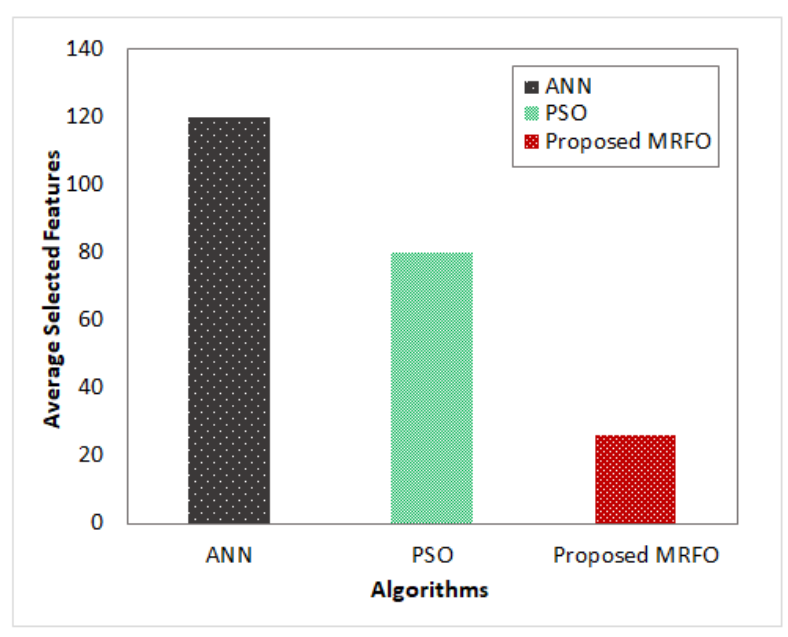

Figure 8 Feature Selection Average Comparison

The performance comparison of proposed model with other models are given in table 3 . Based on the number of features, precision and recall values are observed and it is clearly visible that proposed model attains better precision values.

Table 3 Performance comparison of proposed model

\begin{tabular}{|c|c|c|c|}
\hline Algorithms & Number of features & Precision & Recall \\
\hline Artificial Neural Network & 120 & 0.9204 & 0.9321 \\
\hline Particle Swarm Optimization & 80 & 0.9435 & 0.9560 \\
\hline Proposed Manta Ray Foraging optimization & 26 & 0.9725 & 0.9689 \\
\hline
\end{tabular}




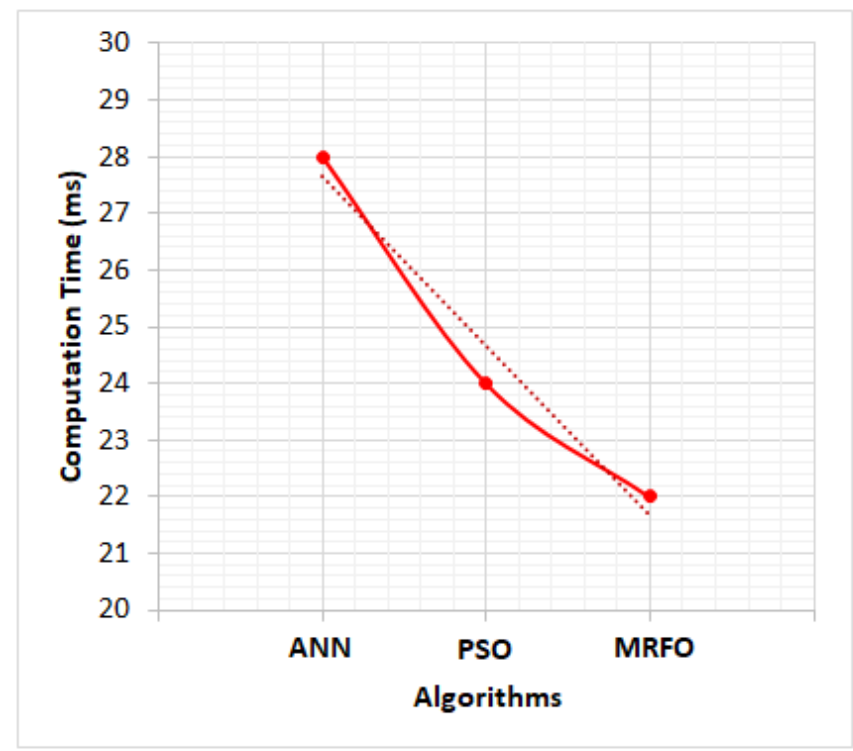

Fig. 9 Computation time comparison

Computation time comparison for all the three models are depicted in figure 9. It is observed that the proposed model consumes minimum time compared to other two models. The observed values are consolidated and obtained as an average computation time and it is compared with linear values.

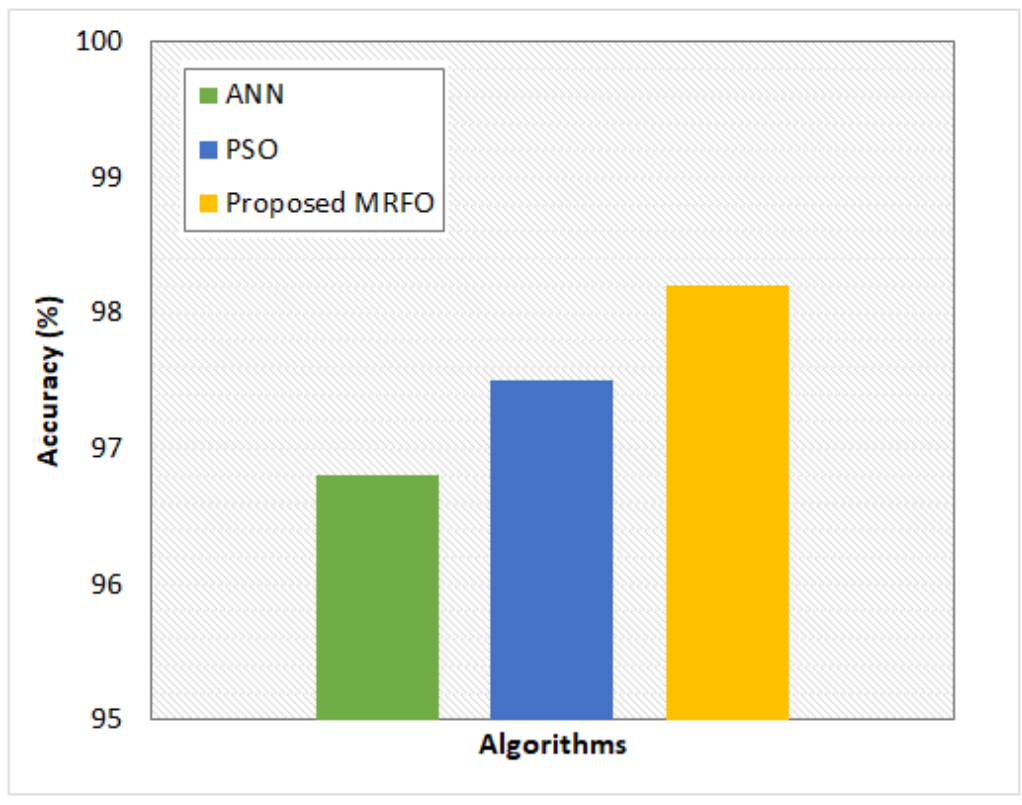

Fig.9 Accuracy comparison

The accuracy comparison of proposed model is depicted in figure 10. The accuracy is calculated based on the output results acquired for the given input image. The number of correct identifications and false identifications are used to calculate the classification accuracy. among the three algorithms, the proposed optimization algorithm attains better accuracy performance of $98.2 \%$ which is much better than other algorithms. 


\section{Conclusion}

A novel optimization model for brain tumor detection is proposed in this research model. By analyzing the issues in conventional models related to brain tumor detection the proposed model is developed using bio inspired optimization algorithm as manta ray foraging optimization algorithm, Based on the foraging process such as chain foraging, cyclone foraging and somersault foraging the proposed model is incorporated and obtains best features to analyze the image. To improve the optimization performance differential evaluation is used which helps to calculate the fitness function and enhances the optimization feature selection process. Convolutional neural network is used as a classifier which further classifies the features and provides better classification results. The experimental model and its performance metrics are compared with artificial neural network and particle swarm optimization model. compared to two algorithms the proposed optimization model attains better accuracy of $98.2 \%$ which is superior than conventional models.

\section{References}

1. Mahmoud Khaled Abd-Ellah, Ali Ismail Awad, Ashraf A. M. Khalaf, Hesham F. A. Hamed (2019). A review on brain tumor diagnosis from MRI images: Practical implications, key achievements, and lessons learned. Magnetic Resonance Imaging, 61, 300-318

2. Devkota, Abeer Alsadoon, P. W. C. Prasad, A. K. Singh, A. Elchouemi (2018). Image Segmentation for Early Stage Brain Tumor Detection using Mathematical Morphological Reconstruction. Procedia Computer Science. 125,115-123

3. Umit Ilhan, Ahmet Ilhan (2017). Brain tumor segmentation based on a new threshold approach. Procedia Computer Science. 120,580-587.

4. Gamal G. N. Geweid, M. A. Elsisy, Osama S. Faragallah, Reza Fazel-Rezai (2019). Efficient tumor detection in medical images using pixel intensity estimation based on nonparametric approach. Expert Systems with Applications. 120, 139-154

5. Sanjeev Kumar, Chetna Dabas, Sunila Godara (2017) Classification of Brain MRI Tumor Images: A Hybrid Approach. Procedia Computer Science. 122, 510-517.

6. Alexander Zotin, Konstantin Simonov, Mikhail Kurako, Yousif Hamad, Svetlana Kirillova (2018). Edge detection in MRI brain tumor images based on fuzzy C-means clustering. Procedia Computer Science. 126,1261-1270

7. Solmaz Abbasi, Farshad Tajeripour (2017). Detection of brain tumor in 3D MRI images using local binary patterns and histogram orientation gradient. Neurocomputing. 219,526-535.

8. Todeschi, C. Bund, H. Cebula, S. Chibbaro, F. Proust (2019). Diagnostic value of fusion of metabolic and structural images for stereotactic biopsy of brain tumors without enhancement after contrast medium injection. Neurochirurgie. 65(6),357-364.

9. Iván Cabria, Iker Gondra (2017). MRI segmentation fusion for brain tumor detection. Information Fusion. 36, 1-9.

10. Bindhu, V. (2019). Biomedical Image Analysis Using Semantic Segmentation. Journal of Innovative Image Processing (JIIP), 1(2), 91-101.

11. Manoharan, S. (2019). Smart Image Processing Algorithm for Text Recognition, Information Extraction and Vocalization for The Visually Challenged. Journal of Innovative Image Processing (JIIP), 1(01), 31-38.

12. ShaoPeng Wang, YuDong Cai(2018). Identification of the functional alteration signatures across different cancer types with support vector machine and feature analysis. Biochimica et Biophysica Acta (BBA) - Molecular Basis of Disease. 1864(6), 2218-2227.

13. Bashar, A. (2019). Survey on Evolving Deep Learning Neural Network Architectures. Journal of Artificial Intelligence, 1(02), 73-82.

14. Alexander Zotin, Konstantin Simonov, Mikhail Kurako, Yousif Hamad, Svetlana Kirillova (2018). Edge detection in MRI brain tumor images based on fuzzy C-means clustering. Procedia Computer Science. $126,1261-1270$.

15. Javaria Amin, Muhammad Sharif, Mudassar Raza, Tanzila Saba, Muhammad Almas Anjum (2019). Brain tumor detection using statistical and machine learning method. Computer Methods and Programs in Biomedicine. 177, 69-79. 
16. Yuanpu Xie, Fuyong Xing, Xiaoshuang Shi, Xiangfei Kong, Lin Yang (2018). Efficient and robust cell detection: A structured regression approach. Medical Image Analysis. 44,245-254.

17. Heba Mohsen, El-Sayed A. El-Dahshan, El-Sayed M. El-Horbaty, Abdel-Badeeh M. Salem (2018). Classification using deep learning neural networks for brain tumors. Future Computing and Informatics Journal. 3(1), 68-71.

18. Saddam Hussain, Syed Muhammad Anwar, Muhammad Majid (2018). Segmentation of glioma tumors in brain using deep convolutional neural network. Neurocomputing. 282,248-261.

19. Philipp Kickingereder, Fabian Isensee, Irada Tursunova, Jens Petersen, Klaus H Maier-Hein (2019). Automated quantitative tumour response assessment of MRI in neuro-oncology with artificial neural networks: a multicentre, retrospective study. The Lancet Oncology. 20(5),728-740.

20. Rajesh Chandra, Kolasani Ramchand H. Rao (2016). Tumor Detection in Brain Using Genetic Algorithm. Procedia Computer Science. 79, 449-457.

21. Muhammad Sharif, Javaria Amin, Mudassar Raza, Mussarat Yasmin, Suresh Chandra Satapathy (2020). An integrated design of particle swarm optimization (PSO) with fusion of features for detection of brain tumor. Pattern Recognition Letters. 129, 150-157.

22. Rabab Hamed M. Aly, Kamel H. Rahouma, Hesham F. A. Hamed (2019). Brain Tumors Diagnosis and Prediction Based on Applying the Learning Metaheuristic Optimization Techniques of Particle Swarm, Ant Colony and Bee Colony. Procedia Computer Science. 163,165-179 